\title{
On carboniferous gigantism and atomic shrinking.
}

\author{
Alain Haraux \\ Sorbonne Université, Université Paris-Diderot SPC, CNRS, INRIA, \\ Laboratoire Jacques-Louis Lions, LJLL, F-75005, Paris, France. \\ e-mail: haraux@ann.jussieu.fr
}




\begin{abstract}
A long standing problem in paleontology is the presence of giant arthropods during the socalled carboniferous era. The same difficulty occurs for the size of plants at the same period. We devise a possible answer to this question entirely different from the theories developed until now, based on the hypothesis that the size of atoms is decaying for very large times and the dimensions of polymers such as chitin and cellulose, containing expansible holes, are decreasing more slowly than rigid materials such as metals, alloys, composite materials and stone. Incidentally, this hypothesis might also give an alternative statement to the expansion of space.
\end{abstract}

Key words: atoms, nucleus, electrons, energy, spectral shift, polymers. 


\section{Introduction}

During the carboniferous era, according to what was found by the specialists, gigantic arthropods such as giant dragonflies, roaches and centipedies (the arthropleura for instance) and many others coexisted with insects of normal size which have been found perfectly conserved inside amber. It is rather difficult to understand how some species of insects could have a size comparable to their present analogs, while some others were larger by almost one order of magnitude, which implies that their volume (and maybe weight, but maybe not according to the subsequent analysis...) should be several hundreds of times larger. This somehow contradicts the fact that species usually stabilize around a certain average size taking account of the fact that similarity laws for the numerous parameters deciding the size are usually different and natural selection leads to convergence of the size. For instance if you multiply the dimensions of a gerris by 10 and keep its density equal to the present one, it will no longer be able to walk on the surface of water.

The currently admitted explanation for this strange phenomenon is the high proportion of oxygen in the air during the carboniferous era (cf. e.g. $[2,4]$ ), which would have given a selective advantage and a capacity to grow much larger in particular to those arthropodes with aquatic larvas, but this does not seem to apply to roaches and does not explain anything about giant plants. It is intriguing to note that (to the author's knowledge) giants arthropodes as well as giant plants did not arrive to us as solid massive objects but we only found their fossilized contours.

The author of the following lines is neither a biologist nor a physicist. He was interested in entomology as a child and was always striken by the paradoxical coexistence of giant insects with normal ones. In the meantime, he lived an entire career as a mathematician and the reason why he recently became interested again in this question is, as we shall see below, somewhat fortuitous.

As a matter of fact, the author started recently to think about the cosmological missing mass enigma and, in the course of his reflexion, was led to study the "redshift" phenomenon from a different perspective. We think that, aside the scientific hypothesis representing the main object of this text, it is not completely useless to explain the very strange succession of events which led to the present proposal.

The plan of this paper is as follows. In Section 2, we explain the connections between three different, at first sight unrelated phenomena. In Section 3, we present some variations of the Rutherford-Bohr atomic model allowing a shriking property of matter. In Section 4 we try to explain the carboniferous gigantism on the basis of atomic shrinking. Sections 5, 6 and 7 are devoted to a discussion of the main text and perspectives of research if the phenomena happen to be confirmed by serious reliable experiments. 


\section{A sudden coincidence of circumstances}

\subsection{A disturbing concrete observation.}

Nothing original, will you tell me. A few days ago, I found in a drawer a small electrical device which had been forgotten there for about 10 years. It was obviously time to change the batteries, but when I took off the old ones, they came out much too easily. Actually there was no contact left between the batteries and the rigid metallic parts which were supposed to drive the current to the appliance. Did the batteries shrink or did the plastic compartment increase in size? Well, let us see with new batteries to decide: the problem turned out to be exactly the same. The compartments of both batteries seem to have been enlarged by something like 0.3 millimeters, and to re-establish the contact I interposed some small pieces of aluminum paper. It would be enough until I find a more elegant solution. But what happenned during that interval of 10 years? There has been no water in the drawer, no overheating, the electrical circuit appliance was left open since to action it you must press a button.

All of a sudden, I remembered that this kind of problems already happened to me several times, and the circumstances were always the same: an electrical device forgotten for 1 or 2 decades, the batteries were cylindrical, the compartments made of plastic, the contacts made of semi-rigid metallic blades. What is going on there? Quite a few persons told me that they experienced the same problem, but thought it is just some kind of aging of the material. After all we are familiar with the idea of "programmed obsolescence". Not very convincing for such a thing.

\subsection{A fancy counter-model for the expansion of universe.}

Of course I could have stopped thinking at this level. But suddenly, I remembered that a few weeks ago, I investigated the fancy idea that the space expansion of universe was an illusion, and the reality might be contraction of matter. I had a look at the web to see if somebody already had this idea, and of course it was the case. On a discussion forum, a philosopher asked the question, and was immediately contradicted by another person having a more developed scientific culture, arguing that in such a case there would be no redshift. This left me undecided, since it was not so clear for me. The idea remained blocked somewhere in my brain with 2 associate questions : how to recover the redshift in the case of matter contraction, and what would be the mechanism involved in contraction? And now, why only now, it was for me absolutely obvious that contraction of matter should be at the atomic level, a reduction of the distance between electrons and nucleons compared with the size of a proton. In such a case, metals should shrink at exactly the same speed as atoms, while materials involving some empty space between atoms could shrink more slowly, for instance polymerized plastic materials.

\subsection{The connection with carboniferous gigantism.}

Immediately after recalling the fancy "atomic shrinking" idea, I remembered the long standing problem of carboniferous gigantism which disturbed me during all these years. It might be that in fact the insects and the plants of the carboniferous period have a normal size with 
respect to their times environment (all atoms being at that time huge compared to now) and, since their skeleton was made of polymerized chitin (respectively cellulose in the case of plants), they would have shrunk more slowly than stone and metal, exactly like the plastic compartments of electric appliances. In this case we clearly end up with huge fossile contours and the paradox disappears. But trying to compute the corresponding enlargment with respect to the environment, starting from the rate computed with the battery-compartment pair, one arrives at an astronomical number, which suggests a more complicated process to explain the enlargment factor of about just one order of magnitude.

\section{Some perturbed Rutherford's models.}

\subsection{Recalling the classical Rutherford-Bohr model.}

The Rutherford model (cf. [5]), which served as a basis for Bohr's model [1] of the hydrogen atom, is based on a corpuscular conception of protons and electrons and the application of Coulomb's law for electrostatic forces. Denoting by $q$ the common absolute value of the charges of proton and electron, the equation of motion, considering the unique proton of the nucleus as the center of coordinates, can be written in the plane of the orbit in complex form. Denoting by $u$ the position of the electron in the complex plane, we have

$$
m u^{\prime \prime}=-\frac{q^{2}}{4 \pi \varepsilon_{0}} \frac{u}{|u|^{3}}
$$

The electron is travelling on a circular orbit

$$
|u(t)|=R
$$

so that the solutions take the form

$$
u(t)=R \exp \left(i \omega\left(t+t_{0}\right)\right.
$$

with

$$
\omega^{2}=\frac{q^{2}}{4 \pi \varepsilon_{0} m R^{3}}
$$

In particular the constant velocity of the electron is

$$
\left|u^{\prime}(t)\right|=v=K R^{-1 / 2}
$$

for some positive constant $K$. This is consistent with the analogous property given by the third Kepler's law for the planets motion driven by the gravitational field of the sun. In the framework of Bohr's modelization, it is assumed that the radius $R$ can take only the values of a sequence of the form $r_{n}=r_{0} n^{2}$ with $n$ a positive integer. In particular the electron cannot "fall" on the nucleus.

Bohr's theory does not explain how the first atoms of matter could appear, even in the simplest case of the hydrogen atom. If an electron is left without initial velocity in the close surrounding of a proton, an atom will never appear since the electron will collide with the proton in a very short time. The problem of the origin of the initial kinetic energy of the electron is left open. Besides, there is no explanation of why some circular orbits would be 
stable and not the others. An attempt to circumvent that problem is quantum mechanics leading to Schrodinger's equation (cf. [6]). For the time being we shall avoid quantum considerations, even though such a framework is presently considered necessary to understand the electromagnetic waves produced by hot matter (and therefore to consider spectral properties and the redshift problematics).

\subsection{A model with damping and tired particles.}

The basic equation (3.1) can be written in the form

$$
u^{\prime \prime}=-\frac{q^{2}}{4 \pi \varepsilon_{0} m} \frac{u}{|u|^{3}}:=-c_{0} \frac{u}{|u|^{3}}
$$

In order to explain a possible exponential decay (with very small decrement) of the atomic radius compared to the size of the proton, we may consider a variant involving a damping mechanism of the form

$$
u^{\prime \prime}+\delta u^{\prime}+c_{0} \frac{u}{|u|^{3}}=0
$$

The origin of the damping has of course to be explained, although damping mechanisms are present everywhere in the real world. Although there might exist decaying solutions of that equation, we did not identify any solution of this equation with an exact exponential decay of $|u(t)|$. The situation changes if we allow the charge $q$ to decay exponentially in time. This leads to the slightly more complicated equation

$$
u^{\prime \prime}+\delta u^{\prime}+c_{0} e^{-\alpha t} \frac{u}{|u|^{3}}=0
$$

for which exponentially decaying solutions can be exhibited under some conditions relating $\delta$ and $\alpha$. In the sequel we shall call $\alpha$ the aging constant.

\subsection{Searching for some explicit exponentially decaying solutions.}

Generalizing the strategy used for the basic equation (3.1) consisting in identifying solutions with constant norms, we look for solutions of the form

$$
u(t)=U e^{-\varepsilon t} e^{i v(t)}
$$

Then

$$
u^{\prime}=\left(-\varepsilon+i v^{\prime}\right) u ; \quad u^{\prime \prime}=\left[i v^{\prime \prime}+\left(-\varepsilon+i v^{\prime}\right)^{2}\right] u ; \quad \frac{u}{|u|^{3}}=U^{-2} e^{3 \varepsilon t} u
$$

and (3.8) reduces to the system

$$
v^{\prime}-2 \varepsilon v^{\prime}+\delta v^{\prime}=\varepsilon^{2}-v^{\prime 2}-\delta \varepsilon+U^{-2} c_{0} e^{(-\alpha+3 \varepsilon) t}=0
$$

The first equation shows an exact exponential variation of $v^{\prime}$ (which might be constant when the decrement is 0 ), and then for the second equation to be consistent we need that either $\varepsilon^{2}=\delta \varepsilon$ or $-\alpha+3 \varepsilon=0$. 


\subsection{Special cases.}

1) For $\varepsilon=\delta$, we find $v^{\prime}=v^{\prime}(0) e^{\delta t}$ and $v^{\prime 2}=U^{-2} c_{0} e^{(-\alpha+3 \delta) t}$, so that $\alpha=\delta$ and $v^{\prime 2}(0)=U^{-2} c_{0}$. This yields a family of anti-clockwise rotating solutions of the form

$$
u_{+}(t)=U e^{-\delta t} \exp \left(i \frac{\sqrt{c_{0}}}{\delta U} e^{\delta t}+i \phi\right)
$$

depending on the two arbitrary parameters $U$ and $\phi$ as in the case of (3.1). We also have clockwise rotating solutions

$$
u_{-}(t)=U e^{-\delta t} \exp \left(-i \frac{\sqrt{c_{0}}}{\delta U} e^{\delta t}+i \phi\right)
$$

These are solution with exponentially fast phase, the kinetic energy of electrons is

$$
\frac{m}{2}\left|u^{\prime}(t)\right|^{2}=\frac{m}{2}\left(\varepsilon^{2}+v^{\prime 2}(t)\right)|u(t)|^{2}=\frac{m}{2}\left(c_{0}+\varepsilon^{2} U^{2} e^{-2 \varepsilon t}\right)
$$

which tends to the constant $\frac{m}{2} c_{0}$ for $t$ large independently of the "initial" radius $U$. Of course the acceleration becomes infinite in norm.

2) For $\varepsilon=\frac{\delta}{2}$, we find $\alpha=3 \delta, v^{\prime \prime}=0$ and $v^{\prime 2}=U^{-2} c_{0}+\frac{\delta^{2}}{4}$. This yields a family of anti-clockwise rotating solutions of the form

$$
u_{+}(t)=U e^{-\frac{\delta}{2} t} \exp \left(i t \sqrt{U^{-2} c_{0}+\frac{\delta^{2}}{4}}+i \phi\right)
$$

depending on the two arbitrary parameters $U$ and $\phi$ as in the case of (3.1). We also have clockwise rotating solutions

$$
u_{-}(t)=U e^{-\frac{\delta}{2} t} \exp \left(-i t \sqrt{U^{-2} c_{0}+\frac{\delta^{2}}{4}}+i \phi\right)
$$

In that case the kinetic energy of electrons, given by

$$
\frac{m}{2}\left|u^{\prime}(t)\right|^{2}=\frac{m}{2}\left(\varepsilon^{2}+v^{\prime 2}(t)\right)|u(t)|^{2}=\frac{m}{2} e^{-\delta t}\left(c_{0}+\frac{\delta^{2}}{2} U^{2}\right)
$$

tends to 0 exponentially. Here also the acceleration becomes infinite in norm.

\subsection{Asymptotics for small values of the dissipation and aging constants.}

It is easy to see on the formulas that with the specific choices of the aging constant (equal to the damping contant or 3 times its value), when $\delta$ tends to zero, we recover the classical circular orbits. The calculation is obvious in the case $\varepsilon=\frac{\delta}{2}$, and in the case $\varepsilon=\delta$ we use the convergence of $\frac{e^{\delta t}-1}{\delta}$ to $t$ as $\delta$ tends to 0 . In that case an infinite shift of the phase is necessary to recover the circular orbits. 


\subsection{The limited interest of these formulas.}

First of all it is not at all natural to require a fixed relation between the damping and aging constants, since these constants are a priori related to quite different, probably unrelated physical phenomena. The above analysis is weak for several reasons

1) We should expect exponentially decaying solutions under an inequality rather than an equality, for instance $\delta \leq \alpha \leq 3 \delta$ is a good guess for the existence of solutions of that type.

2) Even if we find decaying solutions in a much broader context, exactly as in the case of Rutherford's model, we have no argument to assert that nature has "chosen" one of the damped trajectories to guide the electrons. If we want our analysis to become convincing, we therefore need to find an explanation by means of a variation from Schrodinger's model rather than Bohr's modelization. This of course promises to be quite difficult and a thorough analysis of the simple ODE model derived above should be done before proceeding towards a refined quantum model.

Somehow the only interest of the above formulas is their existence, which might guide further research.

\section{A process explaining carboniferous gigantism.}

Compared with the above calculations which raise many difficult questions, explaining carboniferous gigantism is a rather easy task. The "giant insects" and also "giant plants" were identified by their traces in sediments which accumulated in some regions which were before covered with water. So most probably the insects and plants were immersed after death and this gave to their polymerized part the time to inflate with comparison to metals or even liquids were atoms are very close to each other. Actually they did not inflate but they contracted more slowly. The experience with the batteries recalled in the first section suggests a logarithmic decrement of about $10^{-3}$ when the time unit is the year for the size of atoms. Present estimates on the time of recycling of plastic materials turn around 1000 years, but when the substance is confined, the phenomenon slows down since the bacteria take time to arrive on the place. In 1000 years the maximum expansion would be a multiplication by e, and in 2000 years by $e^{2} \sim 8$. After the sediments surround the fossile constrained by its inflated size (compared to hard objects), the fossile itself stops growing and essentially disappears, since after that millions of years elapsed. We are left with a fossilized silhouette which will look between 5 and ten times its real size when we discover it.

\section{Questions for future research.}

First of all the "atomic contraction" conjectured on the basis of concrete everyday observation should be confirmed. The only way seems to be comparison of the dimensional behavior of several materials. By using sophisticated measurement apparatus it should be possible to evaluate the size evolution of materials compared to a chosen unit (which is supposed to shrink!) without waiting for years. The experiments should however be done carefully, keeping a constant temperature since the alleged evolution in one month is comparable to the variation of 
length under a temperature difference of centigrade 10 degrees. This circumstance can actually explain why the phenomenon may have remained hidden (plastic materials are recent, the differential contraction of large pieces of metal with respect to less stable material structures may have been confused with the effect of temperature variation.

Only after this kind of experimental verifications it will become interesting to develop the theory. If it is the case, a difficult program is waiting for us. Even the case of basic ODE is not obvious to master, this being related to the singularity of Coulomb's potential. Then in the framework of quantum mechanics, we should face very serious difficulties even for the simplest case of the hydrogen.

\section{$6 \quad$ Main weak points of the model}

It is a bit too much to speak of a "model" when so many basic questions are not solved. But there are even worse points that it would be dishonest to hide here.

1) If our atoms shrink by a factor 2 every 1000 years, our world should disappear very soon. Because in 20000 years the electrons would reach the nuclei! Therefore we must imagine that a kind of barrier prevents this as it seems to be the case for gravitational forces.

2) The redshift has to be explained. In the past the atoms were larger with an exponential growth when we go backwards in time. We might imagine that the wavelength of electromagnetic radiations increase with the size of atoms, but Hubble's law then requires that the wavelength should be a logarithmic function of the radius of atoms. We have no idea of how such a phenomenon might take place.

3) Last but not the least. Depending on when the shrinking phenomenon started, the exponential growth of atoms in the distant past leads to absurd conclusions. Although the size of atoms does not affect the total mass of planets and stars, beyond a certain size we end up with interpenetration of the planets and the sun, and much before that the dynamics of the solar system becomes difficult to understand...

This might explain why we did not find an exact exponential behaviour even for the solutions of our simple ODEs except for very special parameter coincidences. Our model, if it contains any truth at all, probably has only a rather local (in time) validity.

\section{Conclusion.}

It is always very difficult to question a theory which has required many years of research of our best specialists worldwide. From the theoretical point of view the difficulties are huge even if at some point we might become certain that the phenomenon is real. If it is the case, there will be many potential applications to technology since we could start understanding quite a few breakdowns which occur here and there when we use sophisticated machines during several years. Even if the theory becomes clear, it will never be simple because the present basic theory of matter is already quite involved. In such a situation, if there is some benefit to gain from the atomic contraction hypothesis, computers will play an essential role to improve our technology and increase the duration of sophisticated equipments associating different kinds of materials. 


\section{References}

[1] N.Bohr, On the Constitution of Atoms and Molecules, Philosophical Magazine, Series 26, vol. 1, (July 1913) , p.1-24.

[2] J.F.Harrison , A.Kaiser and J.M. VandenBrooks ; Atmospheric oxygen level and the evolution of insect body size. Proceedings of the Royal Society B. 277(2010):1937-1946. doi:10.1098/rspb.2010.0001

[3] E.Hubble, M.L. Humason, The Velocity-Distance Relation among Extra-Galactic Nebulae, Astrophysical Journal, vol. 74, p. 43.

[4] Ryssa Parks; An Overview of Hypotheses and Supporting Evidence Regarding Drivers of Insect Gigantism in the Permo-Carboniferous, Western Washington University, Spring 2020 .

[5] E. Rutherford; The Scattering of $\alpha$ and $\beta$ Particles by Matter and the Structure of the Atom, E. Rutherford, F.R.S.* Philosophical Magazine, Series 6, vol. 21 May 1911, p. 669-688

[6] E. Schrodinger; An Undulatory Theory of the Mechanics of Atoms and Molecules, Phys. Rev., vol. 28, no 6, decembre 1926, p. 1049-1070 (DOI 10.1103/PhysRev.28.1049) 